\title{
Electrolyte disorders and aging: risk factors for delirium in patients undergoing orthopedic surgeries
}

\author{
Li-Hong Wang ${ }^{1}$, Dong-Juan $\mathrm{Xu}^{2}$, Xian-Jiao $\mathrm{Wei}^{3}$, Hao-Teng Chang ${ }^{4,5}$ and Guo-Hong Xu ${ }^{1 *}$ (D)
}

\begin{abstract}
Background: At present, the exact mechanism of postoperative delirium has not been elucidated. The purpose of this study was to analyze the incidence of delirium in patients undergoing orthopedic surgeries and to explore possible related factors.

Methods: This is a retrospective study. We used 582 patients who had undergone orthopedic surgery between January 2011 and December 2014. The surgeries consisted of 155 cases of internal fixation for intertrochanteric fracture (IFIF), 128 cases of femoral head replacement (FHR), 169 cases of total hip arthroplasty (THA) and 130 cases of total knee arthroplasty (TKA). Among the 582 patients, 75 developed postoperative delirium (an incidence of 12.9\%). The demographics of the patients, which included age, gender, operation duration and blood loss, were statistically analyzed with univariate logistic regression analysis and then multivariate logistic regression. To investigate the influences of different electrolytes disorders for postoperative delirium, the Chi-square test was used.
\end{abstract}

Results: Multivariate logistic regression analysis indicated that postoperative delirium incidence in patients aged $70-79$ years and in patients aged $\geq 80$ years was higher than that in patients aged $<70$ years, odds ratio (OR) values were 6.33 and 26.37, respectively. In addition, the incidence of postoperative delirium in the group of patients with electrolyte disorders was higher than that in the normal group $(O R, 2.38)$. There were statistically significant differences between the delirium group and the non-delirium group in the incidences of the sodium and calcium disorders.

Conclusions: Aging and postoperative electrolyte disorders (hyponatremia and hypocalcemia) are risk factors for postoperative delirium in patients undergoing orthopedic surgeries.

Keywords: Orthopedic surgery, Postoperative delirium, Age-related risk factor, Electrolyte disorders

\section{Background}

Delirium is the most common mental disorder encountered in older patients. However, it is difficult to diagnose because of no unified diagnostic methods and crypticity of postoperative delirium. In different hospital units, the incidence of delirium varied from $10 \%$ in emergency departments [1] to $70 \%$ in intensive care units [2]. The higher figures were associated with frail patients or those with complex surgeries [3]. Postoperative delirium is an acute central nervous system dysfunction after surgical stress

\footnotetext{
* Correspondence: xgh316@163.com

1Department of Orthopedics, Dongyang People's Hospital, Wenzhou Medical University, 60 Wuning West Road, 322100 Dongyang, People's Republic of China

Full list of author information is available at the end of the article
}

that can include clinical features such as acute and nonspecific disturbance of consciousness, attention, cognitive ability and sleep-wake cycles [4]. In orthopedic wards, previous studies indicated that delirium most often occurs after surgeries for hip fracture or hip replacement $[5,6]$. Recently, however, Fineberg et al. [7] and Chung et al. [8] reported postoperative delirium in patients with spine and knee replacement surgeries. This suggested that postoperative delirium may be a common phenomenon in patients undergoing orthopedic surgeries. Postoperative delirium leads to a delay in recovery, extended hospitalization and increased medical costs. It can also lead to bedsores and fall-related fractures. These complications 
can substantially affect the patient's rehabilitation process and quality of life $[9,10]$.

At present, there is no standard procedure for the prevention and treatment of postoperative delirium. Gleason reported that donepezil was effective for the treatment of postoperative delirium [11], but in contrast, the study of Sampson et al. found that donepezil could not significantly reduce the incidence of postoperative delirium or shorten the resulting period of hospitalization [12]. Lowdose haloperidol and olanzapine are probably effective for the treatment of postoperative delirium but not for its prevention [13]. Theoretically, early prevention based on etiology can reduce the incidence of postoperative delirium or may reduce the harm of its effects. However, the etiology and the mechanisms of postoperative delirium are still unclear [14]. Several previous studies have shown that fluid/electrolyte disorders are closely related to postoperative delirium $[7,15,16]$, but the influence of different electrolytes on postoperative delirium remains controversial.

In this study, we retrospectively selected 582 patients who had undergone one of four different orthopedic surgeries, including internal fixation for intertrochanteric fracture (IFIF), femoral head replacement (FHR), total hip arthroplasty (THA) and total knee arthroplasty (TKA). Using logistic regression analyses, we looked for specific risk factors among this population and investigated the mechanism of postoperative delirium. In addition, we tried to find clinical evidence for the prevention and treatment of postoperative delirium.

\section{Methods}

\section{Ethical considerations}

Ethical approval was obtained for this retrospective study from the Internal Review Board of Dongyang People's Hospital. According to the decision of the Internal Review Board, informed consent was not necessary for this retrospective chart review.

\section{Study design and participants}

As a retrospective study, the information described in this paper was obtained from medical chart review. Between January 2011 and December 2014, 695 individuals who underwent one of four orthopedic surgeries (IFIF, FHR, THA and TKA) were enrolled. Among the 695 cases, 113 were excluded because of 20 cases of emergency operation or simultaneous bilateral surgery; 56 cases with mental illness, psychiatric drugs or preoperative cognitive impairment such as dementia; 14 cases with severe vision or hearing disorders who could not complete the cognitive function tests; 11 cases with cerebral infarction or cerebral hemorrhage and 12 cases who were lost to follow-up or for whom the follow-up data were not complete. Thus, a total of 582 cases, including 155 cases of IFIF, 128 cases of FHR, 169 cases of THA and 130 cases of TKA, were finally analyzed in this study.

The 582 cases were divided into two groups: a nondelirium group and a delirium group. The non-delirium group comprised 507 cases and the delirium group comprised 75 cases. According to the medical chart review, a senior psychiatry physician had independently made a diagnosis decision based on the DSM-IV-TR criteria [17] and proposed a treatment plan for ever delirium cases.

\section{Outcome evaluation}

Demographic data including age, gender and blood type were collected. Perioperative factors including duration of hospitalization, type of surgery, anesthesia, preoperative hospitalization, preoperative comorbidities, preoperative and postoperative albumin, preoperative and postoperative hemoglobin, postoperative electrolyte disorders, blood loss, operation time, postoperative intensive care unit (ICU) care and blood transfusion were evaluated through a retrospective chart review. In this study, the preoperative comorbidities were limited to diabetes, chronic obstructive pulmonary disease, pulmonary infections, hypertension and cardiac dysfunction, and the postoperative electrolyte disorders were limited to the higher or lower level than normal for sodium, potassium, chlorine, calcium (Table 1). Clinical laboratory data including the level of albumin and hemoglobin were measured preoperatively and 1 day postoperatively, sodium, potassium, calcium and chlorine were measured 1 day postoperatively. In addition, perioperative blood loss was measured based on hemoglobin levels and weight.

\section{Statistical analysis}

Continuous variables are presented as the mean \pm standard derivation (SD), and a Student's $t$-test was used to determine whether there was a significant difference between the two groups (Table 2). Continuous variables with significant differences were transformed into dummy variables. To examine the effect of demographic and perioperative factors on the development of postoperative delirium, univariate logistic regression was employed first to identify the effective, correlated items, followed by multivariate logistic regression analyses with Enter Method in SPSS. To investigate the influences of different electrolytes disorders for postoperative delirium, the Chi-square test was used for the comparison of categorical variables (Table 5).

Table 1 Electrolyte disorders

\begin{tabular}{lll}
\hline & Disorder $(\mathrm{mmol} / \mathrm{L})$ & Normal $(\mathrm{mmol} / \mathrm{L})$ \\
\hline Sodium & $<135 \mathrm{OR}>145$ & $135 \sim 145$ \\
Potassium & $<3.5 \mathrm{OR}>5.5$ & $3.5 \sim 5.5$ \\
Chlorine & $<95 \mathrm{OR}>105$ & $95 \sim 105$ \\
Calcium & $<2.25 \mathrm{OR}>2.58$ & $2.25 \sim 2.58$ \\
\hline
\end{tabular}


Table 2 Statistical analysis of continuous variables between the delirium group and the non-delirium group

\begin{tabular}{|c|c|c|c|c|}
\hline & Delirium & Non-delirium & $t^{a}$ & $P$-value \\
\hline Cases & 75 & 507 & - & - \\
\hline Duration of hospitalization (d) & $21.25 \pm 8.45$ & $20.85 \pm 8.34$ & 0.42 & 0.68 \\
\hline Preoperative Albumin (g/L) & $38.23 \pm 3.34$ & $39.99 \pm 4.37$ & -3.38 & 0.00 \\
\hline Postoperative Albumin (g/L) & $30.12 \pm 4.46$ & $31.49 \pm 3.78$ & -1.89 & 0.07 \\
\hline Preoperative Hemoglobin $(\mathrm{g} / \mathrm{L})$ & $114.98 \pm 14.56$ & $125.18 \pm 18.32$ & -3.72 & 0.00 \\
\hline Postoperative Hemoglobin (g/L) & $102.21 \pm 15.34$ & $106.98 \pm 17.84$ & -1.60 & 0.14 \\
\hline
\end{tabular}

${ }^{\mathrm{a}} \mathrm{A}$ Student's $t$-test was used to analyze these variables

All statistics were performed with SPSS version 18.0, and the level of significance was set at $P<0.05$.

\section{Results}

\section{Incidence and onset of delirium}

Among the 582 cases of individuals undergoing orthopedic surgery, there were 75 cases of postoperative delirium, an incidence of $12.9 \%$. The onset time of postoperative delirium ranged from $7 \mathrm{~h}$ to a maximum of 16 days after the operation, with an average of 2.8 days postoperation. Most delirium patients showed improvement within 1 week of treatment using haloperidol and regaining electrolyte balance. No mortality during hospitalization occurred because of the postoperative delirium. The incidences of postoperative delirium varied among patients with different surgeries: $17.4 \%$ (27/155) for IFIF, 25\% (32/128) for FHR, 5.9\% $(10 / 169)$ for THA and $4.6 \%$ (6/130) for TKA.

\section{Student's t-test for the continuous variables}

In the comparison with the non-delirium group, patients with postoperative delirium had significantly lower levels of preoperative albumin and hemoglobin $(P<0.01$, Table 2$)$. Preoperative albumin and hemoglobin were then transformed into dummy variables (Table 3 ) and were used in the univariate logistic regression analysis.

\section{Univariate logistic regression analysis}

To carry out a univariate logistic regression analysis, postoperative delirium was set as the dependent variable, and the independent variables consisted of 14 factors including preoperative albumin, preoperative hemoglobin, age, gender, blood type, type of surgery, anesthesia, preoperative hospitalization, preoperative comorbidities, postoperative electrolyte disorders, blood loss, operation time, postoperative ICU care and blood transfusion (Table 3). Univariate logistic regression analysis showed that eight factors were associated with postoperative delirium including age, type of surgery, preoperative hospitalization, preoperative albumin, preoperative hemoglobin, postoperative electrolyte disorders, operation time and postoperative ICU care $(P<0.05$, Table 3$)$.

\section{Multivariate logistic regression analysis}

The eight factors that were associated with delirium based on the results shown in Table 3 were then used in a multivariate logistic regression analysis. This analysis indicated that aging and postoperative electrolyte disorders were the risk factors for postoperative delirium in patients undergoing orthopedic surgeries (Table 4). The incidences of postoperative delirium in the group of individuals aged 70-79 and in the group of individuals $\geq 80$ years old were higher than that in the group of individuals aged $<70$. The incidence of postoperative delirium in individuals with electrolyte disorders was higher than that in individuals with normal electrolyte control (Table 4).

\section{The influence of different electrolytes for postoperative delirium}

The analysis of electrolytes indicated that there were statistically significant differences between the two groups in the incidences of the sodium and calcium disorders (Table 5). All of the postoperative calcium and sodium disorders were hypocalcemia and hyponatremia, respectively except two cases with hypernatremia.

\section{Discussion}

Postoperative delirium is a common phenomenon in older individuals, and it causes recovery delay, extended hospitalization and increases in medical costs. It can have a notable effect on an individual's quality of life. Many previous studies have tried to explain the etiology and the mechanisms of postoperative delirium $[14,18,19]$. However, there is no standard procedure for the prevention or treatment of postoperative delirium. In this study, we showed that aging and postoperative electrolyte disorders were risk factors for postoperative delirium in patients undergoing orthopedic surgeries.

The reported incidence of postoperative delirium varies widely. We speculate that a possible reason for this variability is that the diagnostic methods for postoperative delirium are not uniform. The incidence of postoperative delirium as previously reported ranges from 3.1 to $62 \%$, with the higher incidence in patients after coronary artery bypass surgery [20] and the lower incidence occurring in patients after TKA [8] and spinal surgery 
Table 3 Univariate logistic regression analysis for postoperative delirium

\begin{tabular}{|c|c|c|c|c|c|c|}
\hline Characteristic & $B^{a}$ & S.E.' & Wald & $P$-value & $\mathrm{OR}^{\mathrm{c}}$ & $95 \% \mathrm{Cl}^{d}$ \\
\hline \multicolumn{7}{|l|}{ Gender } \\
\hline Male & - & - & - & - & 1.000 & - \\
\hline Female & -0.053 & 0.256 & 0.043 & 0.835 & 0.948 & $(0.574,1.566)$ \\
\hline Age (years) & & & 38.093 & $<0.001$ & & \\
\hline$<70$ & - & - & - & - & 1.000 & - \\
\hline $70-79$ & 2.709 & 0.738 & 13.478 & $<0.001$ & 15.007 & $(3.534,63.724)$ \\
\hline$\geq 80$ & 3.819 & 0.733 & 27.126 & $<0.001$ & 45.540 & $(10.822,191.637)$ \\
\hline Blood type & & & 4.949 & 0.176 & & \\
\hline A & - & - & - & - & 1.000 & - \\
\hline B & -0.234 & 0.330 & 0.500 & 0.479 & 0.792 & $(0.414,1.513)$ \\
\hline$A B$ & -1.070 & 0.511 & 4.383 & 0.036 & 0.343 & $(0.126,0.934)$ \\
\hline O & -0.020 & 0.304 & 0.004 & 0.947 & 0.980 & $(0.540,1.779)$ \\
\hline Type of surgery & & & 29.940 & $<0.001$ & & \\
\hline IFIF & - & - & - & - & 1.000 & - \\
\hline FHR & 0.458 & 0.294 & 2.420 & 0.120 & 1.580 & $(0.888,2.813)$ \\
\hline THA & -1.210 & 0.389 & 9.689 & 0.002 & 0.298 & $(0.139,0.639)$ \\
\hline TKA & -1.472 & 0.469 & 9.872 & 0.002 & 0.229 & $(0.092,0.575)$ \\
\hline \multicolumn{7}{|l|}{ Anesthesia } \\
\hline General anesthesia & - & - & - & - & 1.000 & - \\
\hline Epidural anesthesia & 0.265 & 0.560 & 0.224 & 0.636 & 1.304 & $(0.435,3.909)$ \\
\hline Preoperative hospitalization (d) & & & 32.560 & $<0.001$ & & \\
\hline $1-3$ & - & - & - & - & 1.000 & - \\
\hline $4-6$ & 1.522 & 0.294 & 26.807 & $<0.001$ & 4.580 & $(2.575,8.148)$ \\
\hline$\geq 7$ & 0.175 & 0.372 & 0.223 & 0.637 & 1.192 & $(0.575,2.469)$ \\
\hline Preoperative comorbidities & & & 6.004 & 0.111 & & \\
\hline 0 & - & - & - & - & 1.000 & - \\
\hline 1 & 0.302 & 0.291 & 1.075 & 0.300 & 1.352 & $(0.764,2.392)$ \\
\hline 2 & 0.162 & 0.357 & 0.205 & 0.650 & 1.176 & $(0.584,2.369)$ \\
\hline$\geq 3$ & 1.107 & 0.459 & 5.825 & 0.016 & 3.026 & $(1.231,7.436)$ \\
\hline Preoperative albumin (g/L) & & & 14.368 & 0.001 & & \\
\hline$<35$ & - & - & - & - & 1.000 & - \\
\hline $35-39$ & 0.000 & 0.341 & 0.000 & 1.000 & 1.000 & $(0.513,1.950)$ \\
\hline$\geq 40$ & -1.061 & 0.379 & 7.838 & 0.005 & 0.346 & $(0.165,0.727)$ \\
\hline \multicolumn{7}{|l|}{ Preoperative hemoglobin ( $\mathrm{g} / \mathrm{L}$ ) } \\
\hline Anemic (male, <120; female, <1 I0) & - & - & - & - & 1.000 & - \\
\hline Normal (male, $\geq 120 ;$ female, $\geq 110$ ) & -0.607 & 0.262 & 5.343 & 0.021 & 0.545 & $(0.326,0.912)$ \\
\hline \multicolumn{7}{|l|}{ Postoperative electrolyte disorders } \\
\hline No & - & - & - & - & 1.000 & - \\
\hline Yes & 0.663 & 0.263 & 6.354 & 0.012 & 1.941 & $(1.159,3.249)$ \\
\hline Blood loss (mL) & & & 3.905 & 0.142 & & \\
\hline$<200$ & - & - & - & - & 1.000 & - \\
\hline 200-399 & -0.260 & 0.264 & 0.971 & 0.324 & 0.771 & $(0.460,1.293)$ \\
\hline$\geq 400$ & -0.866 & 0.456 & 3.606 & 0.058 & 0.421 & $(0.172,1.028)$ \\
\hline
\end{tabular}


Table 3 Univariate logistic regression analysis for postoperative delirium (Continued)

\begin{tabular}{|c|c|c|c|c|c|c|}
\hline Operation time (min) & & & 19.298 & $<0.001$ & & \\
\hline$<60$ & - & - & - & - & 1.000 & - \\
\hline $60-89$ & -0.345 & 0.333 & 1.074 & 0.300 & 0.708 & $(0.369,1.360)$ \\
\hline$\geq 90$ & -1.666 & 0.417 & 15.963 & $<0.001$ & 0.189 & $(0.084,0.428)$ \\
\hline \multicolumn{7}{|l|}{ Postoperative ICU care } \\
\hline No & - & - & - & - & 1.000 & - \\
\hline Yes & 1.005 & 0.256 & 15.443 & $<0.001$ & 2.731 & $(1.655,4.507)$ \\
\hline Blood transfusion $(\mathrm{mL})$ & & & 5.618 & 0.060 & & \\
\hline$<600$ & - & - & - & - & 1.000 & - \\
\hline 600-1199 & 0.666 & 0.281 & 5.618 & 0.018 & 1.947 & $(1.122,3.379)$ \\
\hline$\geq 1200$ & 0.290 & 0.333 & 0.758 & 0.384 & 1.336 & $(0.696,2.564)$ \\
\hline
\end{tabular}

${ }^{\mathrm{a}}$ Regression coefficient

${ }^{\mathrm{b}}$ Standard error

'Odds ratio

${ }^{\mathrm{d}} 95 \%$ confidence interval

[21]. We hypothesize that these differences may be related to diagnostic methods, diagnostic accuracy, sample size and types of surgery. At present, many different diagnostic methods for postoperative delirium have been used. The Confusion Assessment Method (CAM), the CAM for the ICU and the Diagnostic and Statistical Manual of Mental Disorders, Fourth Edition are the most often used diagnostic methods [18]; however, diagnostic criteria are not uniform. In addition, the variability in the individual who assesses delirium-psychiatrists, nurses, or the researchers-could also lead to the perception of different incidences. We believe that it is very important to have a senior psychiatry physician independently make the diagnostic decision. Furthermore, unlike most past research, the present study broadly investigated the incidence of postoperative delirium after four types of common orthopedic surgery, not just a single type of surgery. All these measurements might be helpful to the accuracy of diagnosis.

Although previous studies reported that many factors might be associated with postoperative delirium, such as

Table 4 Multivariate logistic regression analysis for postoperative delirium

\begin{tabular}{lllllll}
\hline & $B^{\text {a }}$ & S.E. & Wald & $P$-value & $\mathrm{OR}^{\mathrm{c}}$ & $95 \% \mathrm{Cl}^{\mathrm{d}}$ \\
\hline Age (years) & & & 24.022 & $<0.001$ & & \\
$<70$ & - & - & - & - & 1.000 & - \\
$70-79$ & 1.845 & 0.788 & 5.477 & 0.019 & 6.328 & $(1.350,29.667)$ \\
$\geq 80$ & 3.272 & 0.808 & 16.414 & $<0.001$ & 26.371 & $(5.415,128.416)$
\end{tabular}

Postoperative electrolyte disorders

$\begin{array}{lllllll}\text { No } & - & - & - & - & 1.000 & - \\ \text { Yes } & 0.865 & 0.367 & 5.556 & 0.018 & 2.376 & (1.157,4.879)\end{array}$

${ }^{\mathrm{a}}$ Regression coefficient

${ }^{\mathrm{b}}$ Standard error

'Odds ratio

${ }^{d} 95 \%$ confidence interval
Alzheimer disease (AD), preoperative hospitalization, anesthesia, diabetes, hemoglobin level, blood loss, operating time and blood transfusion $[5,8,21]$, aging was the factor most often noted in the risk prediction models [18]. Our investigation supports this result. Although the role of postoperative electrolyte disorders in postoperative delirium is still debatable $[15,22,23]$, the presence of an electrolyte disorders or an abnormal electrolyte channel is associated with many neuropsychiatric disorders including AD [24], dementia [25] and depression [26]. Cisternas et al. found that an increased potassium intake can improve cognitive performance and might be important in the prevention of AD onset [27]. Cherbuin's study confirmed that higher intakes of potassium, calcium and magnesium are associated with a reduced risk of developing vascular dementia [28]. Therefore, we have reasons to propose that an electrolyte disorders may be a risk factor for some psychiatric disorders, including postoperative delirium.

Several previous studies have shown that fluid/electrolyte disorders are closely related to postoperative delirium $[7,15,16]$, but the influence of different electrolytes on postoperative delirium remains controversial. Some studies have suggested that a disturbance in potassium or sodium levels might be a factor in postoperative delirium [16, 29], but Caplan's study [19] found that significantly lower serum levels of magnesium and phosphate, not potassium, were associated with delirium. Thus, the exact mechanism by which electrolyte disorders contribute to the occurrence of postoperative delirium is unclear. According to the delirium guideline [30], an electrolyte disorder is a very plausible risk factor for postoperative delirium, and a disturbance in sodium or potassium levels is usually associated with a disorder of body fluids, including hypotonic or hyperosmotic dehydration. A lower or higher level of potassium can occur in combination with 
Table 5 The influence of different electrolytes disorders for postoperative delirium

\begin{tabular}{llllll}
\hline & Delirium & Non-delirium & $P$-value & $\mathrm{OR}^{\mathrm{a}}$ & - \\
\hline Cases & 75 & 507 & - & - & - \\
Potassium disorder & $8(10.7 \%)$ & $27(5.3 \%)$ & 0.07 & 2.123 & $(0.926,4.865)$ \\
Sodium disorder & $20(26.7 \%)$ & $54(10.7 \%)$ & 0.00 & 3.051 & $(1.700,5.472)$ \\
Chlorine disorder & $3(4.0 \%)$ & $53(10.5 \%)$ & 0.08 & 0.375 & $(0.109,1.173)$ \\
Calcium disorder & $42(56.0 \%)$ & $198(39.1 \%)$ & 0.01 & 1.986 & $(1.217,3.241)$ \\
\hline
\end{tabular}

${ }^{\mathrm{a}}$ Odds ratio

${ }^{\mathrm{b}} 95 \%$ confidence interval

metabolic alkalosis or microcirculation disorders, and these can lead to symptoms such as depression, apathy, fatigue, drowsiness, confusion or even coma. Fineberg et al. [7] found that fluid/electrolyte disorders increase the risk of postoperative delirium in patients undergoing lumbar spine surgery. In the present study, we found that the risk of postoperative delirium in patients with electrolyte disturbances was 2.38 times that of individuals with normal electrolytes. Among them, sodium and calcium disorders, especially hyponatremia and hypocalcemia, played an important role in the occurrence of postoperative delirium. Similar to previous studies, we also found that a disturbance in sodium level was a very important risk factor for postoperative delirium. We also found the effect of hypocalcemia on postoperative delirium, which was less studied on postoperative delirium [31]. Therefore, the mechanism of hypocalcemia on postoperative delirium remains unclear. We believe that an effective balance of fluid and electrolytes is important for the prevention of postoperative delirium, which underscores the importance of perioperative management in elderly patients to reduce or avoid the occurrence of postoperative delirium.

\section{Limitations}

This study has some limitations. First, although the phenomenon of postoperative delirium is universal, we have selected four common types of orthopedic surgery, and thus the research results probably cannot explain the incidence of delirium across all types of orthopedic surgery. Second, because this was a retrospective study, case selection could not comply with the principle of randomization. Third, we acknowledge that delirium is hard to be recognized. Although we have carefully checked medical records, we realize that mis-diagnosis might be present. Finally, many factors may be related to postoperative delirium; in this study, not all related factors were included, such as pain [32].

\section{Conclusion}

In conclusion, the present study investigated the risk factors of postoperative delirium following four types of orthopedic surgery. Aging and postoperative electrolyte disorders (hyponatremia and hypocalcemia) were independent risk factors for postoperative delirium in patients undergoing these surgeries. Although the pathogenesis is not fully understood, postoperative delirium is considered a preventable disorder. Surgeons should actively identify risk factors and strengthen the perioperative management of patients to avoid the occurrence of postoperative delirium.

\section{Abbreviations}

FHR: Femoral head replacement; IFIF: Internal fixation for intertrochanteric fracture; THA: Total hip arthroplasty; TKA: Total knee arthroplasty

\section{Acknowledgments}

The authors wish to thank Ying-jun Xu in Medical Records Department of Dongyang People's Hospital for her help with this study.

\section{Funding}

This research was supported by the Health and Family Planning Commission of Zhejiang Province, China (2011KYB152, awarded to Li-hong Wang).

\section{Availability of data and materials}

The data supporting the conclusions of this study is included within the article. Because of linked with personal information, additional data are not available for online access.

\section{Authors' contributions}

LHW and GHX developed the study concept, and LHW wrote the initial draft DJX contributed to the diagnosis and treatment. HTC was involved in the design of this study and contributed to the draft and revisions of the manuscript. XJW performed the data extraction and data analyses. GHX provided interpretations of results. All co-authors made substantial contributions to the paper and approved the final manuscript.

\section{Competing interests}

The authors declare that they have no competing interests.

Consent for publication

Not applicable.

\section{Ethics approval and consent to participate}

Ethical approval (DYPH-2011-KYB152) was obtained for this retrospective study from the Internal Review Board of Dongyang People's Hospital. According to the decision of the Internal Review Board, informed consent was not necessary for this retrospective chart review.

\section{Author details}

${ }^{1}$ Department of Orthopedics, Dongyang People's Hospital, Wenzhou Medical University, 60 Wuning West Road, 322100 Dongyang, People's Republic of China. ${ }^{2}$ Department of Psychiatry, Dongyang People's Hospital, Wenzhou Medical University, Dongyang, People's Republic of China. ${ }^{3}$ Department of Medical Records, Dongyang People's Hospital, Wenzhou Medical University, Dongyang, People's Republic of China. ${ }^{4}$ Graduate Institute of Biomedical Science, College of Medicine, China Medical University, Taichung City 40402, Taiwan. ${ }^{5}$ Department of Computer Science and Information Engineering, Asia University, Taichung City 41354, Taiwan. 
Received: 23 April 2016 Accepted: 15 November 2016

Published online: 23 November 2016

\section{References}

1. Elie M, Rousseau F, Cole M, Primeau F, McCusker J, Bellavance F. Prevalence and detection of delirium in elderly emergency department patients. CMAJ. 2000;163(8):977-81.

2. McNicoll L, Pisani MA, Zhang Y, Ely EW, Siegel MD, Inouye SK. Delirium in the intensive care unit: occurrence and clinical course in older patients. J Am Geriatr Soc. 2003;51(5):591-8.

3. Dyer CB, Ashton CM, Teasdale TA. Postoperative delirium: a review of 80 primary data-collection studies. Arch Intern Med. 1995;155(5):461-5.

4. Silverstein $\mathrm{JH}$, Timberger $\mathrm{M}$, Reich DL, Uysal S. Central nervous system dysfunction after noncardiac surgery and anesthesia in the elderly. Anesthesiology. 2007;106(3):622-8.

5. Juliebo V, Bjoro K, Krogseth M, Skovlund E, Ranhoff AH, Wyller TB. Risk factors for preoperative and postoperative delirium in elderly patients with hip fracture. J Am Geriatr Soc. 2009;57(8):1354-61.

6. Jain FA, Brooks 3rd JO, Larsen KA, Kelly SE, Bode RH, Sweeney GA, Stern TA. Individual risk profiles for postoperative delirium after joint replacement surgery. Psychosomatics. 2011;52(5):410-6.

7. Fineberg SJ, Nandyala SV, Marquez-Lara A, Oglesby M, Patel AA, Singh K Incidence and risk factors for postoperative delirium after lumbar spine surgery. Spine. 2013;38(20):1790-6.

8. Chung KS, Lee JK, Park JS, Choi CH. Risk factors of delirium in patients undergoing total knee arthroplasty. Arch Gerontol Geriatr. 2015;60(3):443-7.

9. Kuwahara M, Yurugi S, Mashiba K, lioka H, Niitsuma K, Noda T. Postoperative delirium in plastic or dermatologic surgery. Eur J Plast Surg. 2008:31(4):171-4.

10. Robinson TN, Raeburn CD, Tran ZV, Angles EM, Brenner LA, Moss M. Postoperative delirium in the elderly: risk factors and outcomes. Ann Surg. 2009;249(1):173-8.

11. Gleason OC. Donepezil for postoperative delirium. Psychosomatics. 2003:44(5):437-8

12. Sampson EL, Raven PR, Ndhlovu PN, Vallance A, Garlick N, Watts J, Blanchard MR, Bruce A, Blizard R, Ritchie CW. A randomized, double-blind, placebo-controlled trial of donepezil hydrochloride (Aricept) for reducing the incidence of postoperative delirium after elective total hip replacement. Int J Geriatr Psychiatry. 2007;22(4):343-9.

13. Boettger S, Jenewein J, Breitbart W. Haloperidol, risperidone, olanzapine and aripiprazole in the management of delirium: A comparison of efficacy, safety, and side effects. Palliat Support Care. 2015;13(4):1079-85.

14. Rudolph JL, Marcantonio ER. Review articles: postoperative delirium: acute change with long-term implications. Anesth Analg. 2011;112(5):1202-11.

15. Zhang WY, Wu WL, Gu JJ, Sun Y, Ye XF, Qiu WJ, Su CQ, Zhang SQ, Ye WQ. Risk factors for postoperative delirium in patients after coronary artery bypass grafting: a prospective cohort study. J Crit Care. 2015:30(3):606-12.

16. Koster S, Oosterveld FG, Hensens AG, Wijma A, van der Palen J. Delirium after cardiac surgery and predictive validity of a risk checklist. Ann Thorac Surg. 2008:86(6):1883-7.

17. Association AP. Diagnostic and statistical manual of mental disorders. 4. Washington, DC: American Psychiatric Association; 2000. Text revision:589-94.

18. van Meenen LC, van Meenen DM, de Rooij SE, ter Riet G. Risk prediction models for postoperative delirium: a systematic review and meta-analysis. J Am Geriatr Soc. 2014;62(12):2383-90.

19. Caplan JP, Chang G. Refeeding syndrome as an iatrogenic cause of delirium: a retrospective pilot study. Psychosomatics. 2010;51(5):419-24.

20. Harmon D, Eustace N, Ghori K, Butler M, O'Callaghan S, O'Donnell A, Moore-Groarke GM, Shorten G. Plasma concentrations of nitric oxide products and cognitive dysfunction following coronary artery bypass surgery. Eur J Anaesthesiol. 2005;22(4):269-76.

21. Gao R, Yang ZZ, Li M, Shi ZC, Fu Q. Probable risk factors for postoperative delirium in patients undergoing spinal surgery. Eur Spine J. 2008;17(11):1531-7.

22. Koster S, Hensens AG, Schuurmans MJ, van der Palen J. Prediction of delirium after cardiac surgery and the use of a risk checklist. Eur J Cardiovasc Nurs. 2013;12(3):284-92.

23. Korevaar JC, van Munster BC, de Rooij SE. Risk factors for delirium in acutely admitted elderly patients: a prospective cohort study. BMC Geriatr. 2005;5:6.

24. Widner B, Leblhuber F, Fuchs D. Abnormal potassium-channel function in platelets in Alzheimer's disease. Lancet. 1999;353(9149):325-6.
25. McKeon A, Marnane M, O'Connell M, Stack JP, Kelly PJ, Lynch T. Potassium channel antibody associated encephalopathy presenting with a frontotemporal dementia like syndrome. Arch Neurol. 2007;64(10):1528-30.

26. MacMillan HL, Gibson JC, Steiner M. Hyponatremia and depression. J Nerv Ment Dis. 1990;178(11):720-2

27. Cisternas $P$, Lindsay CB, Salazar P, Silva-Alvarez C, Retamales RM, Serrano FG, Vio $C P$, Inestrosa NC. The increased potassium intake improves cognitive performance and attenuates histopathological markers in a model of Alzheimer's disease. Biochim Biophys Acta. 2015;1852(12):2630-44.

28. Cherbuin N. Higher dietary intakes of potassium, calcium and magnesium are associated with a reduced risk of developing vascular dementia. Evid Based Ment Health. 2013;16(1):26

29. Shiiba M, Takei M, Nakatsuru M, Bukawa H, Yokoe H, Uzawa K, Tanzawa H. Clinical observations of postoperative delirium after surgery for oral carcinoma. Int J Oral Maxillofac Surg. 2009:38(6):661-5.

30. van der Mast RC, Huyse FJ, Rosier PF. [Guideline 'Delirium']. Ned Tijdschr Geneeskd. 2005;149(19):1027-32.

31. Aldemir M, Ozen $\mathrm{S}$, Kara $\mathrm{IH}$, Sir A, Bac B. Predisposing factors for delirium in the surgical intensive care unit. Crit Care. 2001;5(5):265-70.

32. Vaurio LE, Sands LP, Wang Y, Mullen EA, Leung JM. Postoperative delirium: the importance of pain and pain management. Anesth Analg. $2006: 102(4) \cdot 1267-73$

\section{Submit your next manuscript to BioMed Central and we will help you at every step:}

- We accept pre-submission inquiries

- Our selector tool helps you to find the most relevant journal

- We provide round the clock customer support

- Convenient online submission

- Thorough peer review

- Inclusion in PubMed and all major indexing services

- Maximum visibility for your research

Submit your manuscript at www.biomedcentral.com/submit
Biomed Central 